\title{
A STEWART PLATFORM AS A FBW FLIGHT CONTROL UNIT
}

\author{
Vasfi Emre ÖMÜRLÜ — İbrahim YILDIZ *
}

\begin{abstract}
A variety of flight control units have been put into realization for navigational purposes of spatially moving vehicles (SMV), which is mostly manipulated by 2 or 3 degrees-of-freedom (DOF) joysticks. Since motion in space consists of three translational motions in forward, side and vertical directions and three rotational motions about these axis; with present joystick interfaces, spatial vehicles has to employ more than one navigational control unit to be able to navigate on all required directions. In this study, a $3 \times 3$ Stewart-Platform-based FBW (Fly-By-Wire) flight control unit with force feedback is presented which will provide single point manipulation of any SMVs along three translational and about three rotational axis. Within the frame of this paper, design, capability and the advantages of the novel system is mentioned. Kinematics of a Stewart Platform (SP) mechanism employed and its motion potentials is presented by simulations and workspace of the system is evaluated. Dynamic analysis by Bond-Graph approach will be mentioned. Mechatronic design of the complete structure is discussed and force reflection capability of the system with simulations is pointed out using stiffness control. Finally, the possible future work of the subject is discussed which may include the feasible solutions of the SP in terms of size and safety when implementing inside a cockpit.
\end{abstract}

K e y w or ds: flight control, stewart platform, spatial motion, force feedback control, bond-graph modeling, workspace analysis

\section{INTRODUCTION}

Stewart Platform related researches have gradually increased since it was first used as a flight simulator in 1965, [1]. In general, these studies are kinematic analysis of the parallel mechanisms (forward/inverse kinematic analysis, [2-6]), dynamic behavior, [7], control, [8-10], design of different simulators, [11, 12], and manufacturing, [4]. Among these, especially, kinematic analysis research for mechanisms resulting simpler mathematical solutions to reduce computation time is very significant.

Inverse/direct dynamics of SP mechanisms is also very important area since it establishes the connection between input force/torque and output positional motion data. Both Newton-Euler [13] and Lagrange Methods, [14], are employed for forward and inverse dynamics solutions by several researchers. Additionally, the BondGraph Method is employed for dynamic modeling of an SP mechanism utilizing energy bonds between elements as in [15]. Workspace and singularity analysis is another research area of these mechanisms and since forward kinematics of parallel mechanisms are problematic, various solutions have been proposed, $[5,16]$.

Stewart Platform based systems are also employed in haptic applications and in surgical simulators, [6, 17]. Although flight and surgical simulator applications of parallel mechanisms are present, SP has not been used as a man-machine interface to manipulate vehicles performing spatial motion. Especially, since linear dc actuators are very fast in response time, their utilization in parallel mechanisms will result in very transparent haptic manmachine interface.
In this study, linear dc motor actuated $3 \times 3 \mathrm{SP}$ as a man-machine interface is proposed as a means of mechatronic design, kinematic analysis and simulation environment to manipulate SMVs allowing force feedback capability so that environmental forces acting on the manipulated vehicle can be reflected to the user. Through the paper, first, suitability of the SP mechanism to manipulate SMVs is discussed. Following the analysis of SP mechanism for kinematics and dynamics, workspace of the system is investigated and the parameters affecting the workspace which are related to the manipulated vehicle are discussed. Then, mechatronic design issues and force control of the mechanism is presented. Lastly, the possible future work of the subject is mentioned.

\section{STEWART PLATFORM AND ITS CAPACITY AS A FLIGHT CONTROL UNIT}

\subsection{SP Mechanism}

Many applications related to physical simulation of spatial motion have been performed since the first emergence of Stewart Platform based parallel mechanisms. The Stewart Platform (SP) is a parallel robotic mechanism, which is mostly used for simulating flight patterns of flying objects and is employed in manufacturing industry where spatial motion is needed for complicated surface machining. It has a moving upper plate and stationary lower plate and six parallel-connected actuator legs attached to these plates which enable the upper plate for spatial motion. In present study, $3 \times 3 \mathrm{SP}$ is used in which six legs are connected to upper platform at three points and are connected to the lower stationary platform at three points as well. Since the moving plate of

* Yildiz Technical University, A309 Yildiz/Besiktas,Istanbul, Turkiye, 34349, omurlu@yildiz.edu.tr 


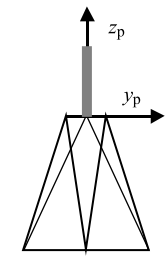

Front

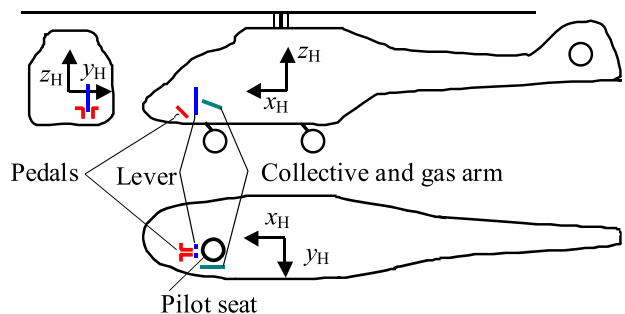

Pilot seat

Fig. 1. Front, up and side views of a helicopter and an SP mechanism as a flight control unit
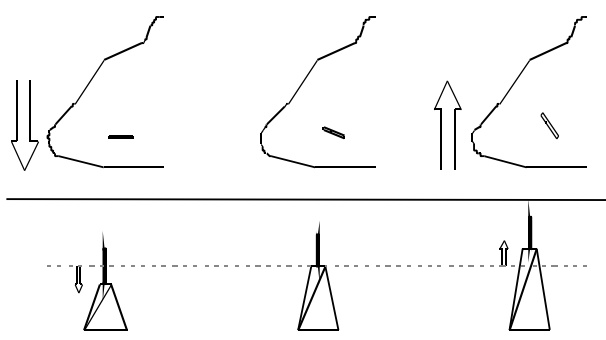

Collective down Helicopter descending

C ollective centered Helicopter hovering

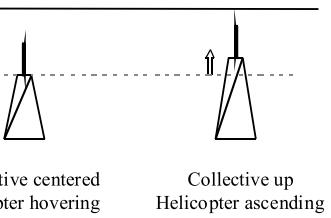

Fig. 2. State of ascending, hovering and descending of a helicopcopter with SP-based flight control

the mechanism is capable of spatial motion, this system can be used to manipulate vehicles in space like a six DOF joystick instead of employing a lower DOF mechanism which engages more than one hand of the pilot. Because during any flight, excessive controls and situations of importance should be considered at once, navigating the vehicle completely with one hand should reserve single unique significance.

\subsection{Spatial Motion Manipulation by an SP}

Pilots generally have to interact with more than one manipulation unit in order to manage the navigation of SMVs. For instance, a lever, a pedal and a collective is employed to control a helicopter, Fig. 1. The collective arm provides ascending and descending motion of the helicopter in z, vertical, direction, Fig. 2. There exists a gas arm on top of the collective in order to provide instantaneous power to the vehicle. Pedals are used for rolling motion around $\mathrm{z}$ axis, Fig. 3, and the lever is for pitch and yaw motion of the helicopter, Fig. 4 .

A standard $3 \times 3 \mathrm{SP}$ mechanism with force feedback, apparently, allows the user to manipulate any SMV. The user employs the lever on the SP to move the mechanism. The motion of the SP is interpreted by an interface in order to obtain desired positional/directional motions of the vehicle. In the pictorial representations on Figs. 1-4, basic motions of the vehicle is shown on a manipulator placed on the left of a helicopter. Instantaneous collective motion is obtained through using the SP.

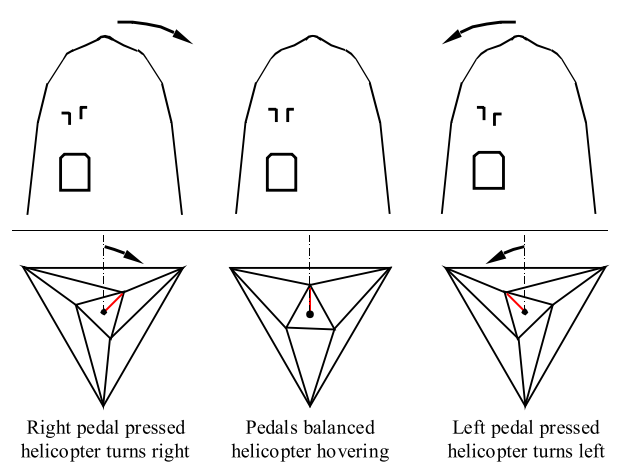

Fig. 3. Roll motion of a helicopter performed by an SP mechanism as a flight control unit

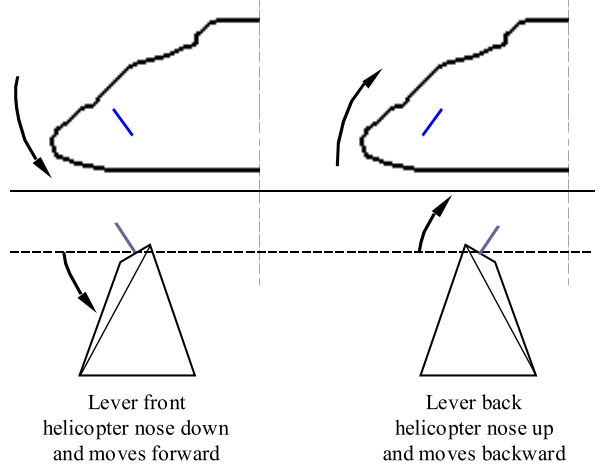

Fig. 4. Pitch and yaw motion of a helicopter by an SP mechanism as a flight control unit

\section{KINEMATICS AND DYNAMICS OF THE 3X3 SP}

An experimental setup of $3 \times 3 \mathrm{SP}$ and the physical model of the SP mechanism is shown in Fig. 5 .

The experimental setup and the model has six linear motors as actuators. Each actuator is attached to the upper platform and the lower platform by spherical joints. Upper part of the SP mechanism performs following motions:

Linear motion along $x_{P}, y_{P}$ and $z_{P}$ axis; $t_{x}, t_{y}, t_{z}$ respectively.

Rotation around $x_{P}, y_{P}$ and $z_{P}$ axis; $\gamma, \vartheta, \nu$ respectively.

It is assumed that:

- Center of gravity of upper platform is located at the origin of the mobile frame.

- Center of gravity of actuators is located about a distance of the $2 / 3$ of the total actuator length from the lower connection point.

- Bearing frictions and gravitational forces are present on the system.

The parallel interaction between actuators causes angular velocities on each leg. 

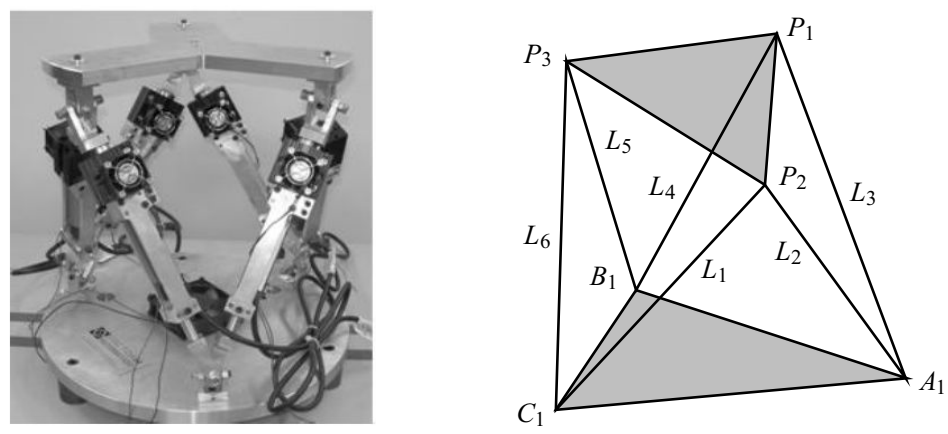

Fig. 5. The $3 \times 3 \mathrm{SP}-$ Experimental Setup and the Physical Model
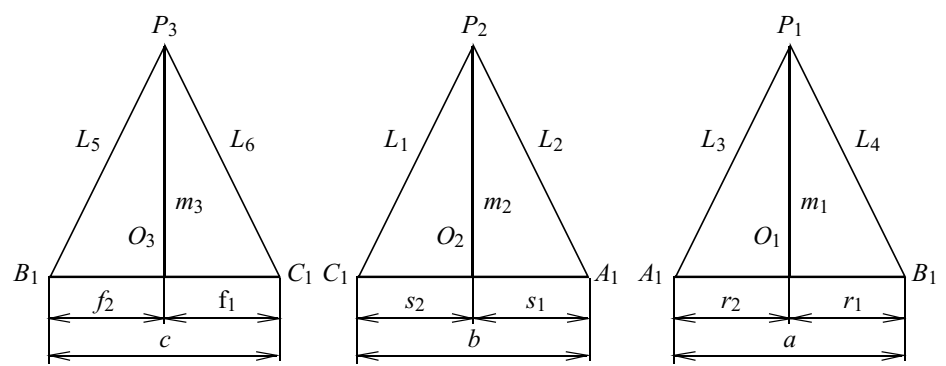

Fig. 6. Positions of the $O_{i}$ points and the $m_{i}$

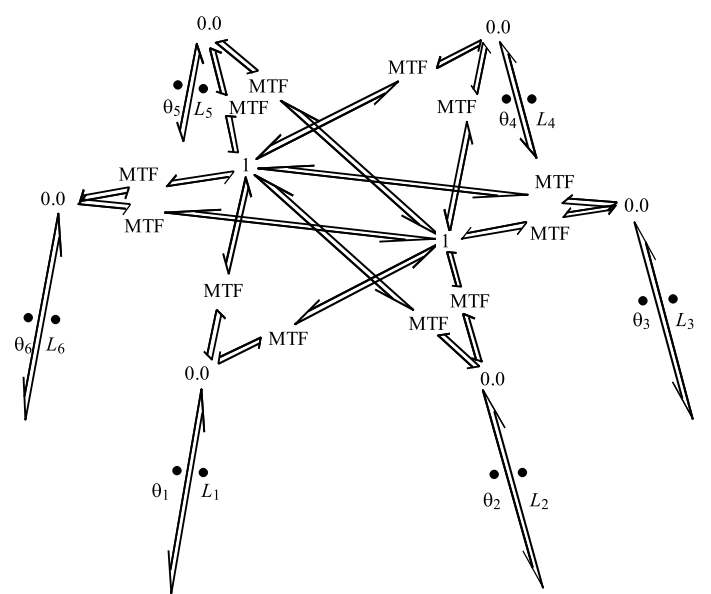

Fig. 7. Complex kinematic vector bond-graph model of the SP mechanism

\subsection{Kinematics of the $3 \times 3 \mathrm{SP}$}

Forward and inverse kinematics of the platform is considered in which the former one is more complicated one for parallel mechanisms where the moving upper platform rotational and directional data should be calculated by the use of leg lengths, $L_{i}$. It is to be assumed that the connection points of the stationary, $B_{i}$, and moving platforms, $P_{i}$, with respect to the reference axis of the lower platform, $T$, and the reference axis of the upper platform, $P$, respectively, are all known.

For inverse kinematics, the position of the connection points of the upper platform, $P_{i}$, needs to be calculated with respect to $T$ using the rotation matrix, $R$, and the position vector, $t$, of the $P$ with respect to $T$.

$$
P_{i}=R p_{i}+t .
$$

Forward kinematics aims to find the $t$ vector and rotations of the upper platform based on the $L_{i}$ values and the boundary conditions of constant edge lengths of the stationary platform. On the plane of each adjacent and connecting leg on $P_{i}, O_{i}$ is the projection point of the upper connection point on the edges of the lower platform and $\phi_{i}$ is the angle between the $m_{i}$ and the lower platform plane. In this calculation, $O_{i}, m_{i}$ and $\omega_{i}$ values are the parameters in the equations where $\omega_{i}$ is the unit vector along $m_{i}$ which depends on $\beta_{i}$ and $\phi_{i}$ angles. $\beta_{i}$ are the angles between the stationary $x$-axis and the line between $T$ and $O_{i}$, Fig. 6 . As a result, three nonlinear equations with three unknowns appear and $\phi_{i}$ are calculated through Bezout matrix. Then, placing the results in previous equations, $P_{i}$ can be found.

Kinematics of the platform is also represented through Bond-Graph technique. Translational and rotational velocities along and about the actuators can be expressed by the terms of upper platform velocities.

In Equation (2) and (3), the angular velocity of upper platform multiplies by the first, second and third columns of $K^{L_{i}}$ and $K^{\theta_{i}}$ same as the linear velocity of upper platform multiplies by the fourth, fifth and sixth columns of $K^{L_{i}}$ and $K^{\theta_{i}}$.

$$
\begin{aligned}
& \dot{L}_{i}=\underbrace{\left[K_{1 . .3}^{L_{i}} K_{4 . .6}^{L_{i}}\right]}_{K^{L_{i}}}\left[\begin{array}{c}
\vec{\omega} \\
\dot{\vec{t}}
\end{array}\right], \\
& \dot{\vec{\theta}}_{i}=\underbrace{\left[K_{1 . .3}^{\theta_{i}} K_{4.66}^{\theta_{i}}\right]}_{K^{\theta_{i}}}\left[\begin{array}{c}
\omega \\
\dot{\vec{t}}
\end{array}\right] .
\end{aligned}
$$




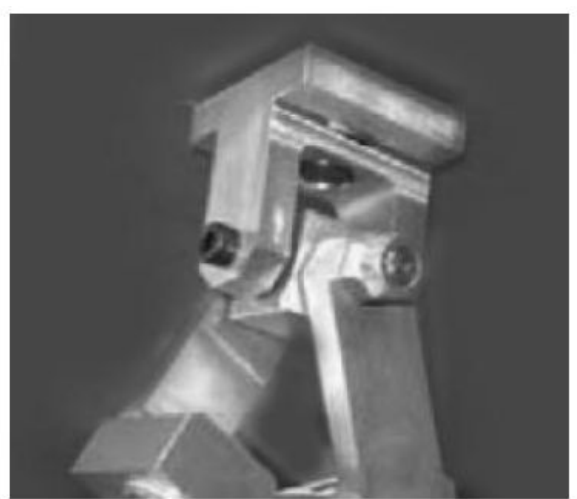

Fig. 8. Spherical joint of SP mechanism

In complete model, each parallel energy ports have 12 energy bonds and each leg has different translational and rotational velocities. A new model is developed to understand the relationship between the kinematic structure of the system and the physical model as in Fig. 7.

\subsection{Dynamics of the $3 \times 3 \mathrm{SP}$ and Bond - Graph Approach}

Attaching the physical elements of the system to the kinematic model, dynamic Bond-Graph model of SP mechanism can be obtained.

When two or more inertial elements are attached to " 1 port, system state variables become dependent and this causes derivative causality problem. This means, independent velocities of these elements becomes dependent velocities and this problem results in an implicit differential equation which may cause numerical problems in solution. This problem can be solved by transforming inertial elements of dependent velocities to the ports of independent velocities. Therefore, inertial elements should be reduced to the ports of independent velocity ports as a virtual inertia matrix and a gyristor element. Dependent velocities, independent velocities and inertial velocities of the system should be defined before reducing the system. Translational and rotational velocity of legs can be chosen as dependent velocities, therefore upper platforms translational and rotational velocities can be chosen as independent velocities. All dependent and independent velocities can be chosen as inertial velocities.

All inertial elements of the system should be defined with a single inertia matrix before obtaining virtual inertial matrix and gyrator element. Inertial elements should be arranged using inertial velocity matrix.

The actuation of the system is based on linear DC actuators which are chosen as legs of the SP mechanism. Each linear motor is mounted to mobile and stationary platform by spherical joints, Fig. 8. The gravitational effect on legs and upper platform are modeled by effort sources, and the actuation inputs of the legs are modeled by effort sources.
Resultant dynamics of the SP mechanism in statespace form may be represented as

$$
\begin{aligned}
& \frac{\mathrm{d}}{\mathrm{d} t}\left[\begin{array}{c}
\omega_{x} \\
\omega_{y} \\
\omega_{z} \\
\dot{t}_{x} \\
\dot{t}_{y} \\
\dot{t}_{z} \\
i_{a_{1}} \\
i_{a_{2}} \\
i_{a_{3}} \\
i_{a_{4}} \\
i_{a_{5}} \\
i_{a_{6}}
\end{array}\right]=\left[\begin{array}{ll}
{[A]_{6 \times 6}} & {[B]_{6 \times 6}} \\
{[C]_{6 \times 6}} & {[D]_{6 \times 6}}
\end{array}\right]\left[\begin{array}{c}
\omega_{x} \\
\omega_{y} \\
\omega_{z} \\
\dot{t}_{x} \\
\dot{t}_{y} \\
\dot{t}_{z} \\
i_{a_{1}} \\
i_{a_{2}} \\
i_{a_{3}} \\
i_{a_{4}} \\
i_{a_{5}} \\
i_{a_{6}}
\end{array}\right] \\
& +\left[\begin{array}{cc}
{\left[\tilde{I}^{-1}\right]_{6 \times 6}} & 0 \\
0 & {\left[L_{a}\right]_{6 \times 6}}
\end{array}\right][U]_{12 \times 1} .
\end{aligned}
$$

Here, the state variables are the translational/rotational velocities of the upper platform and the motor currents. U represents inputs to the system which includes gravitational effects and the excitation voltages to DC motors.

\subsection{Workspace Analysis}

Workspace of a kinematic mechanism is the reachable max volume of the center of moving platform, $\mathrm{P}$ and several factors affect the workspace of the system: a) actuator, leg, lengths has direct effect on the workspace of the mechanism, b) actuator diameter, especially, is employed in collision conditions and the effective diameter is calculated from $r_{k i} \sin \phi_{i}$ in which $r_{k}$ is one of the two leg diameters and the $\phi_{i}$ is angle between the leg and the vertical axis, [18], c) angular position capability of the joints is one of the most important limitations of the workspace, d) singularity points [19,20].

Since related equations are in simpler form, inverse kinematics equations are utilized in order to determine the workspace capability of the SP mechanism. Possible workspace is divided into certain number of points and legs are actuated in simulations for these target points so that reachable ones can be identified after applying boundary conditions.

In order to determine the workspace of the mechanism for certain parameters, a versatile analysis should be considered. Though overall affecting parameters of the workspace for an SP is 48, [21], assuming a workspace consisting of only points, no rotations of the upper platform, no positional data about these workspace points, and circular pattern of upper and lower platform connection locations, $r_{i}$ and $R_{i}$ respectively, these parameters may be reduced to $r$ and $R$,radius of mobile and stationary platform connection points to the motors, [22].

With the use of vectorial relations of $T$ and $P$, an elliptical equation which relates $r$ and $R$, is obtained that can be used for numerical workspace analysis. Since required motion capabilities of any SMV in different directions as a means of positioning and speeding may vary, 

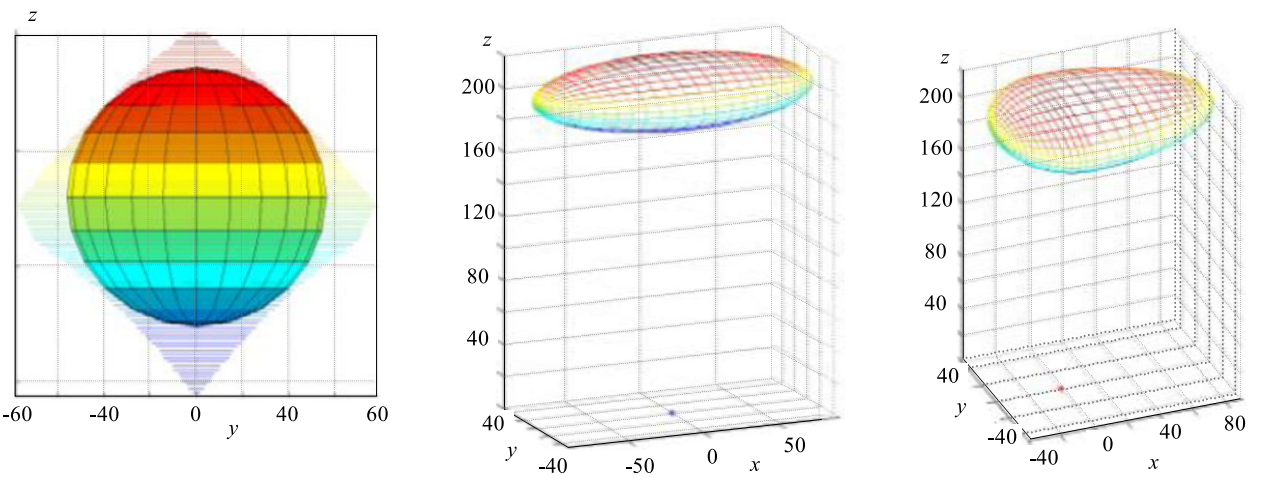

Fig. 9. Spherical, ellipsoid and egg-like workspace to be fit into the overall workspace of the SP

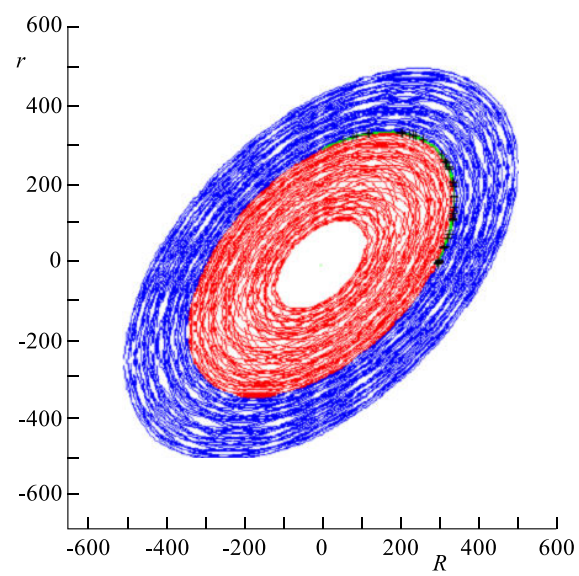

Fig. 10. The collection of $r$ and $R$ values (+) which satisfies the spherical workspace radius of $56 \mathrm{~mm}$

a three types of workspaces is approached, Fig. 9. On the left side of the figure, it is explicitly shown that the spherical workspace to be reached is fit into the overall workspace of the SP. The solution of mentioned elliptical equation provides the design parameters of $r$ and $R$ for the required workspace. For example, Fig. 10 gives the possible combination of $r$ and $R$ radius which will result in a spherical workspace of $56 \mathrm{~mm}$ radius.

\section{MECHATRONIC DESIGN AND FORCE CONTROL}

\subsection{The System}

As seen on Fig. 11, the system consists of a lever (A), a six axis force/torque transducer (B) of ATI nano25, a motion control card interface (C) of NI UMI-7774, a six axis motion control card (D) of NI PCI-7356, a PC, linear motor drivers of E210-VF (E), and a $3 \times 3$ SP. Lever is rigidly assembled to the force/torque transducer while the transducer is also rigidly assembled to the Stewart Platform so that force/torque interaction between the lever and the SP is fully measurable. The force/torque transducer, linear motor drivers and the motion control cards data signal cables are connected to motion control card interface.
The forces/torques that are applied to the lever by the user to manipulate the SMV, are sensed by the force/torque transducer, (three axis of force and three axis of torque) and converted to the analogue voltage data ( \pm 10 volts) separately for each axis. Then this data is sent to the motion control cards analogue inputs. The motion control cards ADCs converts the analogue data to sixteen bits of digital data. The software that is written for this system gets the digital data and converts it to numerical values of forces and torques. By this way the software interprets the pilots control commands (or more understably, in which direction in space s/he intends moving/rotating to) and calculates the values for the motion control card to produce the analogue voltage output which also takes into account of the disturbances like wind and inertial forces. The software uses inverse kinematics to calculate the legs lengths.

Since linear dc actuator drivers is run under force/torque mode, the \pm 10 Volts of signals is sent to linear motors drivers to convert the data to actuating forces by controlling the motors current in which full control capability is transferred to the control designer comparing to velocity mode where internal velocity control algorithms of the drivers are heavily used. The encoder data of selectable resolutions $(1 \mathrm{~mm} \rightarrow 1 \mu \mathrm{m})$ from the auxiliary encoder output of the driver is used for positional feedback purposes. The software developed in Visual Basic utilizes this data for closed loop control of the system.

By these complicated control mechanisms, the pilot feels the external forces through the lever which is acting on the vehicle like controlling with former controllers unless the air vehicle does not navigate in ideal surroundings.

\subsection{Force Control by Stiffness Control Assisting the User}

A stiffness control algorithm for force feedback purposes, [24], is applied to Sim-mechanics based dynamic model of $3 \times 3$ SP manipulator. As placed in the real system mentioned in previous section, it is assumed that there is a force/torque sensor mounted over the moving platform which measures the force/torque interaction between the user and the moving platform. Also, the stiffness of the environment (in this case user constitutes the 


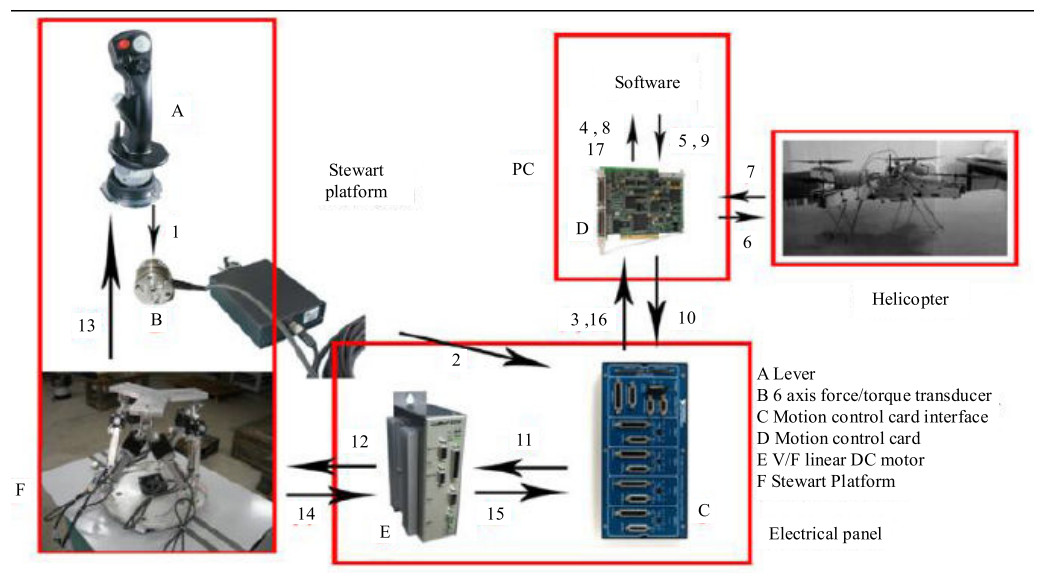

Fig. 11. General components of the overall Stewart Platfom mechatronic system

environment) is given by KFP, Fig. 12. As a result, force data is transformed into position data using basic linear spring equation of $F=K x$. After deriving platform positions, lengths of each leg are obtained from inverse kinematics method. Classical proportional integral derivate (PID) type controller is applied to each leg, separately.

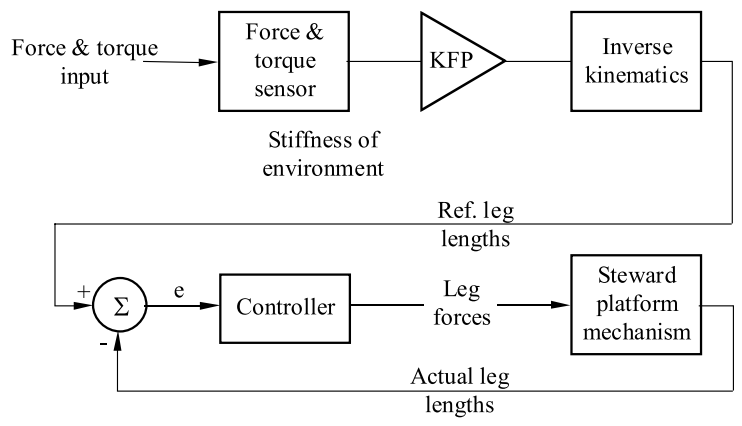

Fig. 12. Stiffness Control of SP Mechanism with Force Feedback where proportional-integral-derivative control is employed

Figure 13 shows the input force and output trajectory. Sinusoidal external forces of $30 \mathrm{~N}$ in magnitude with 90 degree phase for each $x$ and $y$ axis is applied to the system and response of the SP mechanism on the left is received. Since, in our application, motion assistance is intended, the user should not feel any forces/torques when using the lever as if $\mathrm{s} /$ he was driving the SMV (through SP mechanism) in free space with no disturbance. Figure 14 shows the error between applied and reaction forces of the manipulator. This figure also indicates that reaction forces of SP Mechanism is almost equal to the applied forces acting on manipulator pointing that close approximation is reached to free space simulation with raw control parameters.

\section{CONCLUSION}

In this study, a diverse use of SP mechanism is introduced in which the potential of spatial motion simulation of the SP mechanism is reflected to the manipulation of SMVs. Though versatile use of SP mechanism is present from manufacturing to spatial motion simulation, manipulating vehicles in space by this mechanism with force feedback has been left untouched. Because of the complexity of the mechanism and of the intended area of usage, various aspects of the mechanism need to be analyzed. Kinematics and resultant workspace analysis is carried out in order to maximize the spherical workspace of the mechanism, in spite of the fact that possible workspaces of different shapes is also suggested depending on the motion capabilities of the vehicle to be manipulated. Dynamic analysis of the system is performed through a non-classical approach, Bond - Graph technique, which directly provides system equations in nonlinear state-space equations giving the designer better chance to reach the control solutions and dynamic analysis. Mechatronic design of the real system is presented in which the overall system components are presented in detail. Since the platform is utilized as a spatial joystick with some certain weight and internal damping of the bearings, first, the moving platform is to be stationed on a mid-point when not used or in auto-pilot, then, moving the upper platform should not require any extra effort for the user, "assisted-user mode. Thus, a stiffness control as a free space simulator is proposed with a classical PID controller and the results of the response of the system as a means of the reflected force to the users hand is provided. Several steps needs to be taken in order to finalize this research, some of which are, kinematically, to determine the workspace requirements of the real cockpit controllers for various SMVs, to investigate different force control algorithms for better force transparency and to search for an optimized mechanical and mechatronical design approach to reach a more feasible solutions that fits into the intended vehicle.

\section{Acknowledgements}

Authors of this research appreciate Mr Seref Naci Engin, Mr İbrahim Beklan Küçükdemiral, and Mr Utku Buyuksahin, for their valuable contributions. This work is supported by The Scientific and Technological Research Council of Turkey (TUBITAK) grant 3501-105M192. 

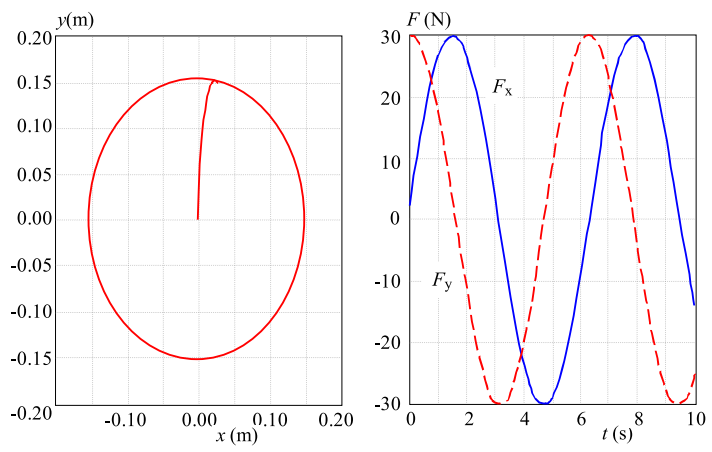

Fig. 13. Output Positional Trajectory and Input Force of Stiffness Error $F(\mathrm{~N}) \quad$ Controller

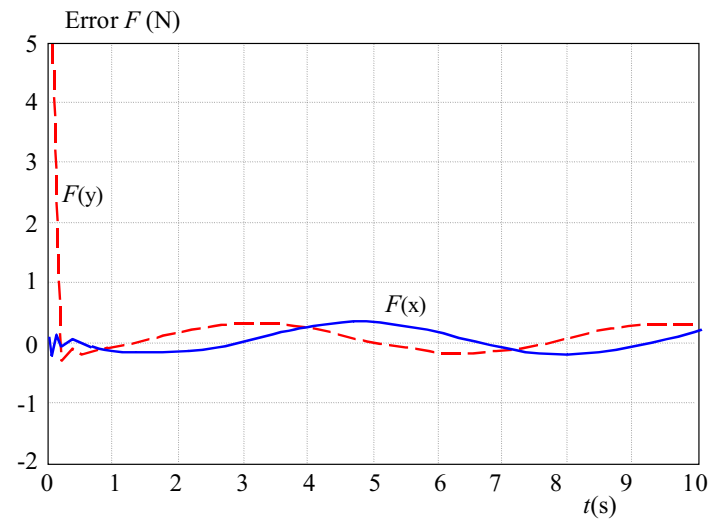

Fig. 14. Error between applied and reaction forces using stiffness control

\section{REFERENCES}

[1] STEWART, D. : A Platform with Six Degrees of Freedom, Proc. Instn. Mech. Engrs 80 (1965), 371-386.

[2] ZANG, C.-SONG, S. : Forward Kinematics of a Class of Parallel (Stewart) Platforms with Closed-Form Solutions, International Conference on Robotics and Automation, 1991.

[3] ZHUANG, H.-YAN, J.-MASORY, O.: Calibration of Stewart Platforms and Other Parallel Manipulators by Minimizing Inverse Kinematic Residuals, Journal of Robotic Systems 15 (1998), 395-405.

[4] HARIB, K.-SRINIVASAN, K. : Kinematic and Dynamic Analysis of Stewart Platform-Based Machine Tool Structures, Robotica 21 (2003), 541-554.

[5] KIM, D.-CHUNG, W.: Analytic Singularity Equation and Analysis of Six-DOF Parallel Manipulators Using Local Structurization Method, IEEE Transactions on Robotics and Automation 15 No. 4 (Aug 1999), 612-622.

[6] STOCCO, L.-SAlCUdEAN, S. E. : A Coarse-Fine Approach to Force-Reflecting Hand Controller Design, IEEE International Conference on Robotics and Automation, Minnesato, April 1996.

[7] JASON GENG, Z.-HAYNES, L. S.: Six Degree-of-Freedom Active Vibration Control Using the Stewart Platforms, IEEE Transactions on Control Systems Technology 2 No. 1 (March 1994), 45-53.

[8] SU, Y. X.-DUAN, B. Y.-ZHENG, C. H.-ZHANG, Y. F.CHEN, G. D.-MI, J. W. : Disturbance-Rejection High-Precision Motion Control of a Stewart Platform, IEEE Transactions on Control Systems Technology 12 No. 3 (May 2004), 364-374.

[9] LEE, S.-SONG, J.-CHOI, W.-HONG, D. : Position Control of a Stewart Platform Using Inverse Dynamics Control with Approximate Dynamics, Mechatronics 13 (2003), 605-619.
[10] LEE, S.-SONG, J.-CHOI, W.-HONG, D. : Controller Design for a Stewart Platform Using Small Workspace Characteristics, IEEE/RSJ International Conference on Intelligent Robots and Systems, Maui-Hawai, USA, Oct 2001, pp. 2184-2189.

[11] WENDLANDT, J. M.-SASTRY, S. S.: Design and Control of a Simplified Stewart Platform for Endoscopy, Proceedings of the $33^{\text {rd }}$ conference on Decision and Control, Lake Buena Vista, FL, Dec 1994, pp. 357-362.

[12] MOON, Y.-KOTA, S.: Design of Compliant Parallel Kinematic Machines, Proceedings of DETC02, Montreal, Canada, Sep 29-Oct 2, 2002, pp. 1-7.

[13] DAsGupta, B.-MRUThyUnjayA, T. S.: Closed-Form Dynamic Equations of the General Stewart Platform Through the Newton-Euler Approach, Mechanism and Machine Theory 33, 993-1012.

[14] LEBRET, G.-LIU, K.-LEWIS, F. L.: Dynamic Analysis and Control of a Stewart Platform Manipulator, Journal of Robotic Systems 10, 629-655.

[15] YILDIZ, I.-—ÖMÜRIÜ, F.L.-SAĞIRLI, A. : Dynamic Modeling of a Generalized Stewart Platform by Bond Graph Method Utilizing a Novel Spatial Visualization Technique, International Review of Mechanical Engineering 2 No. 5 (2008).

[16] PERNKOPF, F.-HUSTY, M.: Workspace Analysis of Stewart-Gough Manipulators Usign Orientation Plots, Proceedings of MUSME, 2002.

[17] KUDOMI, S.-YAMADA, H.-MUTO, T. : Development of a Hydraulic Master-Slave System for Tele-Robotics, Proc. of $1^{\text {st }}$ FPNI-PhD Symp., Hamburg, Germany, 2000, pp. 467-474.

[18] WAN, Y.-WANG, S.: Kinematics Analysis and Simulation System Realization of Stewart Platform Manipulator, The Forth Internatioanal Conference on Control and Automation (ICCA03), Montreal, Kanada, 10-12 July 2003, pp. 780-784.

[19] KIM, D.-CHUNG, W.: Analytic Singularity Equation and Analysis of Six-DOF Parallel Manipulators Using Local Structurization Method, IEEE Transactions on Robotics and $\mathrm{Au}-$ tomation 15 (Aug 1999), 612-622.

[20] TSAI, K. Y.-LEE, T. K.-HUANG, K. D.: Determining the Workspace Boundary of 6-DOF Parallel Manipulators, Robotica 24 (2006), 605-611.

[21] MERLET, J. P.: Designing a Parallel Manipulator for a Specific Workspace, INRIA Sophia-Antipolis, Report No: 2527, April 1995.

[22] MERLET, J. P. : Designing a Parallel Manipulator for a Specific Workspace, The International Journal of Robotics Reearch 16 (Aug 1997), 545-556.

[23] ZENG, G.-HEMAMI, A. : An Overview of Robot Force Control, Robotica 15 (1997), 473-482.

Received 31 August 2009

Vasfi Emre Ömürlü was born in Istanbul/Turkiye in 1969. He received his MSc degree in Yıldız Technical University of Istanbul/Turkiye about vehicle dynamics in 1993 and completed his $\mathrm{PhD}$ studies "Surgical robotics" in Ohio State University of Columbus, Ohio/USA in 2002. He is currently working on parallel robotic manipulators, autonomous aerial vehicles and automation systems, and is the vice chair of recently established Mechatronic Engineering Department of Yıldız Technical University.

İbrahim Yıldız was born in Konya/Turkiye on 26th of August. He received his MSc degree in Yıldız Technical University of Istanbul/Turkiye about the topic of dynamics of $3 \times 3$ stewart platform mechanisms on year 2007. He has just received his Ph.D. degree on the force control of parallel manipulators. He is currently working on force control of parallel mechanisms, biomechanics and aerial vehicles. He is currently the research/teaching assistant in Yıldız Technical University. 